\title{
BERICHT ÜBER DEN VERLAUF DER TAGUNG
}

\author{
Viorel IoniṬA
}

\section{Die Vorbereitung}

Die theologische Aus- und Weiterbildung ist mehr und mehr ein gemeinsames Anliegen der Kirchen in Europa. In dieser Hinsicht wollen sich die Kirchen verpflichten, durch die Charta Oecumenica, die am 22. April 2001 im Rahmen der Europäischen Ökumenischen Begegnung in Strassburg unterschrieben wurde, „ökumenische Offenheit und Zusammenarbeit in der christlichen Erziehung, in der theologischen Aus- und Fortbildung sowie auch in der Forschung zu fördern".

Gleichzeitig wurde die Bedeutung der theologischen Fakultäten und Bildungsstätten für die Ökumene während der letzten Jahre von verschiedenen ökumenischen Organisationen und Initiativen immer wieder betont. Die Grazer KatholischTheologische Fakultät organisierte eine Begegnung von Delegierten der orthodoxen, katholischen, evangelischen, islamischen und jüdischen Fakultäten der Nachbarländer Österreichs im Jahre 1993 in Graz. Dort wurde die ökumenische Zusammenarbeit der theologischen Fakultäten und als langfristiges Ziel die Schaffung einer ökumenischen „,Konferenz der theologischen Fakultäten und Hochschulen Europas " empfohlen. Auf Einladung des Ökumenischen Rates der Kirchen hatte 1996 in Oslo eine internationale Tagung zur theologischen Ausbildung im ökumenischen Kontext stattgefunden. Die Konferenz Europäischer Kirchen (KEK) hat in Zusammenarbeit mit dem Diakonischen Werk der Evangelischen Kirche in Deutschland (EKD) ein Seminar zur Frage der ökumenischen Aus- und Weiterbildung im Jahre 1999 in Duràu, Rumänien, veranstaltet.

Der Reformierte Weltbund (RWB) hat zwei Konsultationen (1994 und 1995) über theologische Aus- und Weiterbildung für die RWB-Mitgliedskirchen in Mittel- und Osteuropa organisiert. Das Ausbildungsreferat des Kirchenamtes der EKD hat seinerseits zwei europäische Konsultationen über Fragen der theologischen Ausbildung in Berlin (1994 und 1995) durchgeführt. Darüber hinaus wurden in den letzten Jahren einige Zusammenschlüsse der theologischen Fakultäten aus verschiedenen Regionen Europas gegründet: z.B. Ost-Mitteleuropäischer Fakultätentag (1997) oder die theologischen Fakultäten von Skandinavien in Verbindung mit dem ökumenischen Kirchenrat im Norden. Hier ist auch auf die Bedeutung der Societas Oecumenica hinzuweisen. 
Durch diese Zusammenarbeit ist eine ganze Reihe von Fragen aufgetaucht, vor allem in Hinblick auf die theologische Aus- und Weiterbildung in einer ökumenischen Perspektive. Unter diesen Fragen sind die Kompatibilität der unterschiedlichen Systeme der theologischen Aus- und Weiterbildung auf europäischer Ebene untereinander, die institutionelle Anbindung, Curricula u.a.m., zu klären. Eine weitere Herausforderung ist die gegenseitige Anerkennung von Studienleistungen und Studienabschlüssen in den verschiedenen Ländern bzw. Kirchen Europas. Ein mehr systematischer Austausch zwischen den europäischen theologischen Fakultäten und Bildungsstätten ist dabei wünschenswert.

Aufgrund dieser Erfahrung hat die KEK die Initiative ergriffen, gemeinsam mit der Katholischen Theologischen Fakultät der Universität Graz und in Zusammenarbeit mit der Evangelischen Kirche in Deutschland und der Europäischen Gesellschaft für Katholische Theologie, eine Konsultation zu organisieren. Auf dieses Ziel hin wurde eine internationale Gruppe mit der Planung dieser Konsultation beauftragt. Zu der Planungsgruppe, die ihre Arbeit im Januar 2000 angefangen hatte, gehörten:

\author{
Prof. Dr. Peter Balla, Ungarn \\ Prof. Dr. Juray BANDY, Slowakei \\ Drs. Gerrit Douma, Holland \\ Rev. Arne J. ERIKSEN, Norwegen \\ Prof. Dr. Grigorios LaRentZaKIS, Österreich \\ Prof. Dr. Gerhard LARCher, Österreich \\ Prof. Dr. Viorel IONITA, KEK \\ Prof. Dr. Anne Kull, Estland \\ Prof. Dr. John Muddiman, England \\ OKR Dr. Günter WASSERBERG, EKD
}

Durch ihre intensive Arbeit hat die Planungsgruppe das Thema „Die Zukunft der Theologie in Europa“"vorgeschlagen wie auch ein Programm entworfen. Diese Konsultation der Theologischen Fakultäten in Europa fand im Bildungshaus Mariatrost, Graz, Österreich, vom 4.-7. Juli 2002 statt. Es nahmen daran mehr als 60 Vertreter der verschiedenen theologischen Fakultäten und Kirchen aus 24 Ländern teil. Dabei waren die anglikanischen, orthodoxen und reformatorischen theologischen Traditionen vertreten. Die theologische Tradition der Freikirchen war in Graz leider sehr schwach vertreten. Arbeitssprachen der Konsultation waren Deutsch und Englisch. 


\section{Die Eröffnung}

Die offizielle Eröffnung der Konsultation fand auf die Einladung der KatholischTheologischen Fakultät der Karl-Franzens-Universität Graz am Donnerstag, 4. Juli um 17 Uhr in der Aula dieser Universität in Anwesenheit der TeilnehmerInnen wie auch vieler Gäste statt. Prof. Dr. Friedrich Zimmermann, Vizerektor der Universität Graz stellte in seinem Grusswort die Universität vor. Prof. Dr. Gerhard LARCHER, Dekan der Katholisch-Theologischen Fakultät der Universität Graz begrüsste alle Anwesenden als Gastgeber und Prof. Dr. Viorel IonITA sprach ein Dankeswort an die Gastgeber im Namen der KEK als Hauptorganisator.

Die drei folgenden Referate zum Hauptthema der Konsultation: „Die Zukunft der Theologie in Europa“ wurden aus dem Blickwinkel ,konfessionell/ökumenisch/pluralistisch“ gehalten. In seinem eröffnenden Beitrag erläuterte Bischof Dr. Egon KaPELLARI von Graz die Begriffe Theologie, pluralistisch, konfessionell und ökumenisch und betonte zum Schluss seiner Anmerkungen, dass die Theologie sich besonders im europäischen Horizont um einen ökumenischen Dialog bemühen muss. Europa, von wo die Kirchenspaltung ausgegangen ist, fuhr Bischof KAPELLARI fort, ,könnte so zu einem Modellkontinent inner-christlichen Friedens gestaltet werden. Und die Ökumene könnte eine ekklesiale und politische Schrittmacherfunktion haben für die religiöse und kulturelle Befriedung des Kontinents, wenn die Christen lernen, mit der neuen und zunehmenden Multikulturalität und Multireligiösität in Europa unter Wahrung der eigenen Identität unbefangen umzugehen."

In dem darauffolgenden Referat identifizierte die Bischöfin Dr. Bärbel WARTENBERG-PotTER aus Lübeck, Deutschland, aufgrund ihrer langjährigen Erfahrung in der theologischen Ausbildung und in der weltweiten Ökumene die wichtigsten Herausforderungen an die theologische Aus- und Weiterbildung in Europa von heute und äusserte einige Wünsche für die zukünftige Arbeit auf diesem Gebiet:

\section{„Die Ausbildungsgänge innerhalb Europas öfnen und kompatibel ma- chen.}

Neben den alten Sprachen eine europäische Sprache verbindlich lernen.

Ein Praktikum, Studienzeit in einer überseeischen Partnerkirche verbringen.

Ökumenisches Lernen ist ein Schlüssel für die Erneuerung der Kirche. Konfessionelle Institutionen öffnen für Studierende anderer Konfessionen, auch für Frauen.

Geschlechtsspezifische Themen in der Ausbildung thematisieren.

Spirituelle und studienmäßige intensivere Begleitung der Studierenden ermöglichen.

Selbstorganisiertes Lernen ermutigen. " 
Zum Schluss der Eröffnungssitzung sprach Michael STAIKos, Metropolit von Austria, Wien, Österreich, über die Bedeutung der theologischen Ausbildung aus der Sicht der Zusammenarbeit zwischen den Theologen und den „Hirten der Kirche“ und betonte dabei, dass das Amt der Lehrer in der Kirche immer eine zentrale Rolle gespielt hat, ,,auch wenn es nicht immer adäquat und gerecht geschätzt wurde. Das Amt des Lehrers in der Kirche hat auch mit dem kirchlichen Lehramt zu tun“.

Metropolit Michael begrüsste zum Schluss die Initiative, eine Konsultation der theologischen Fakultäten in Europa zu organisieren und äusserte seine Hoffnung, dass ,heute ein Grundstein gelegt wird für eine intensive Fortsetzung der Zusammenarbeit auf Europa-Ebene, unabhängig von Konfession und Region. Langfristig könnte ich mir auch eine strukturelle Zusammenarbeit unserer Fakultäten vorstellen".

In seiner Zusammenfassung über die einführenden Beiträge unterstrich Prof. Dr. John Muddiman die wertvolle Leistung der drei kirchlichen Würdenträger und betonte, dass eine grosse Herausforderung an die Theologie in Europa heute durch die Spannung zwischen konfessionell, ökumenisch und pluralistisch dargestellt wird. Prof. Muddiman meinte, dass man sich diese drei Aspekte wie drei sich überschneidende Kreise vorstellen kann, die in einer gewissen Spannung stehen und daraus entstehen viele kritische Fragen.

\section{Die Hauptreferate}

Das Hauptthema der Konsultation wurde im Verlauf der Tagung durch vier Hauptreferate vertieft. Die ersten zwei zur Frage: ,Die Zukunft der Theologie in Europa: Theologie/Religionswissenschaft", wurden von Prof. Dr. Konstantin DeliKostantis, Athen, Griechenland, bzw. von Prof. Dr. Sven Erik Brodd, Uppsala, Schweden, gehalten.

In der Einführung seines Referates, unter dem Titel: ,,Theologie und Religionswissenschaft. Sinn und Unsinn eines Konflikts“, sprach Prof. Delikostantis von einer Orientierungs- und Identitätskrise der Theologie, ,,der wir unsere gemeinsame Anstrengung entgegenstellen müssen". Weiter betrachtete der orthodoxe Referent die Frage der Theologie ,als Wissenschaft im Haus der Wissenschaften“ als westliche Tradition, und betonte, dass aus orthodoxer Sicht, die Religionswissenschaft auf keinen Fall die Theologie ersetzen kann. Religionswissenschaft und Theologie können und sollen sich ergänzen.

Nach einigen wichtigen Überlegungen zur Frage der Beziehung zwischen Theologie und Religionswissenschaft in Griechenland machte Prof. Delikostantis die Bemerkung, dass eine Religionswissenschaft, welche den Direktiven und den Interessen der Kirche und der Theologie folgt, ,, und deren Ertrag an dem Kriterium, was theologisch annehmbar und bequem ist gemessen würde, wäre auch für die Theologie letztlich bedeutungslos. Somit wäre aber die Theologie von einer sehr wichtigen Erkenntnisquelle in Sachen Religion abgetrennt". 
Zum Schluss fasste der Referent seine Überlegungen zusammen: „Die Theologie muss ständig uns daran erinnern, dass keine Kultur, keine Zivilisation eine endgültige Lösung des menschlichen Dilemmas, keine letzte Bleibe für den ,konstituiv wurzellosen "Menschen darstellt, auch nicht die wissenschaftlichtechnische Zivilisation mit ihren vielen Wundern. Der Mensch transzendiert die Kultur; sein ,Wunsch ganz zu sein '; seine ,Sehnsucht nach der Heimat ' kann nicht, auch durch die höchste unter den Kulturen, gestillt werden."

Der zweite Referent zum Thema ,Die Zukunft der Theologie“ im Spannungsfeld „Theologie/Religionswissenschaft“, Prof. Dr. Sven Erik BRODD, ging von der Feststellung aus, dass mehr als alle anderen Akademiker und Akademikerinnen wahrscheinlich die Theologen und Theologinnen an den Universitäten überall in Europa sich damit beschäftigt haben, ,nach ihrer Rolle unter den Disziplinen, ihrer Existenzberechtigung, ihren Theoriesystemen und schliesslich auch nach ihrem eigenen Vorverständnis, also ihrem Glauben bzw. nicht vorhandenen Glauben zu fragen. Dafür gibt es mehrere Gründe, die sowohl intern sind, also von den theologischen Fakultäten selbst kommen, als auch extern sind, also beeinflusst von den Debatten im kulturellen, religiösen und politischen Umfeld."

Eine weitere interessante Bemerkung von Prof. BRODD war hinsichtlich der Bedeutung der nichtchristlichen Theologie an der Universität. „In früheren Zeiten ist das Studium der nichtchristlichen Religionen als eine Stufe zur Vorbereitung der Theologie, zur Vermittlung theologischer Vorkenntnisse verstanden worden oder auch als konkreter und klar abgegrenzter Bereich innerhalb oder auch ausserhalb der theologischen Fakultäten, der oftmals Religionswissenschaft oder Geschichte der Religion(en) genannt wurde. Übrigens scheint es immer noch so zu sein, dass nichtchristliche Theologen recht wenig Interesse an der Universitätstheologie zeigen. Die historischen Gründe dafür liegen auf der Hand, aber es könnte doch erwartet werden, dass sich dies allmählich ändert. Hier liegt eine Herausforderung nicht nur an die theologischen Fakultäten, sondern auch an die Universität selbst.“

Der Referent aus Schweden behandelte weiter die folgenden Fragen: 1. Die Organisation der Universitätstheologie und die theologischen Fakultäten; 2. Die „wissenschaftliche“ Universität und die Theologie; 3. Religionswissenschaftliche Studien und Universitätstheologie; 4. Die Zersplitterung der religionswissenschaftlichen Studien; 5. Der normative Charakter der Universitätstheologie in der gelehrten Gesellschaft; 6. Der Beitrag der theologischen Fakultät zur Universität; 7. Der Unterschied zwischen Theologie studieren und theologischen Studien; 8. Religionswissenschaft und Theologie - einige abschliessende Bemerkungen.

Nach seinen aussführlichen Betrachtungen, wodurch er zwischen dem Studium der Theologie oder der Religionswissenschaft, das notwendig ist, und den theologischen Studien, die grundlegend sind, unterschied, kam Prof. BRODD zu dem gleichen Ergebnis wie Prof. Delikostantis und zwar, dass „,Religionswissenschaft oder , religious studies "die Theologie an der Universität nicht ersetzen kann“. 
Ausgehend von der Kritik in Schweden, ,wo der Universitätstheologie von Zeit zu Zeit vorgeworfen wird, dass sie sich an eine eingebildete Idee von der Wertneutralität der Universität angepasst habe“, meinte der Prof. aus Uppsala, dass diese Kritik nicht auf die theologischen Fakultäten allein zu beschränken sei, ,,sondern es geht um die Universität an sich, ihre Isolierung und ihre Grenzen. Vielleicht haben die theologischen Fakultäten dabei trotz allem eine Rolle zu spielen und können ein mutiges Beispiel davon geben, wie Wissen und Weisheit zum Nutzen der Menschheit bewahrt, geschaffen und vermittelt werden können. “

Die letzten zwei Hauptreferate haben das Thema:,Die Zukunft der Theologie in Europa " im Spannungsfeld ,,berufliche/akademische Ausbildung " betrachtet und wurden von Prof. Dr. Duncan ForRester, Edinburgh, Schottland bzw. von Prof. Dr. Michael BeINTKer, Münster, Deutschland gehalten.

Prof. Duncan Forrester gab seinem Beitrag den Titel: „Die Zukunft der Theologie: Die Berufung und das ,Akademische " in der theologischen Ausbildung" Nach einigen kritischen Überlegungen über die alte Vision der Theologie als „Königin der Wissenschaften“ und der praktischen Theologie als „Krone des Universitätsstudiums“, setzte sich der Referent mit heutigen Herausforderungen an die Theologie durch die Universitäten auseinander. Der Referent meinte, dass in der zersplitterten postmodernen Universität viel Energie auf die Abgrenzung zwischen den Disziplinen verschwendet wird, ,was manchmal absurde Konsequenzen zeitigt. Dabei sollte es ein zentrales Anliegen der Theologie sein, Gemeinschaft zu stiften und die Kommunikation über Grenzen und Schranken hinweg zu fördern."

Für den Professor aus Edinburgh ist die wahrhaftige Theologie Lobpreis Gottes, oder Theologie in der Gegenwart Gottes und zur Ehre Gottes zu betrachten. Diese Art von Theologie ist nach Prof. ForRESTER tatsächlich möglich an einer modernen säkularen Universität, ,und wenn sie verwirklicht wird, ist sie die beste intellektuelle und spirituelle Ausbildung für Pfarrerinnen und Pfarrer und für die Nachfolgegemeinschaft, die in einer radikal pluralen und säkularen Gesellschaft leben und arbeiten wird, um ihre verschiedenen Berufungen und Ämter auszuüben. Die säkulare pluralistische Universität ist mit allen ihren Problemen sicherlich der beste Ort, um ein Verständnis für das dienende und prophetische Amt zu entwickeln, das für die Welt der Zukunft relevant sein wird."

Schliesslich kam Prof. ForRester auf die ökumenische Ausbildung zu sprechen, die aus seiner Sicht für die Zukunft der Kirche Jesu Christi von allergrößter Wichtigkeit ist. „Die Freundschaften und Vertrauensgrundlagen, die dadurch zwischen Studierenden unterschiedlicher Konfessionen und Traditionen möglich werden, können dazu beitragen, die ökumenische Situation , von Grund auf" zu verwandeln. Dies kann in ökumenischen Seminaren oder im Konsortium konfessioneller Seminare organisiert werden. Besonders viel aber spricht dafür, wenn die Studierenden tief im laufenden intellektuellen Leben einer Universität verwurzelt und eng in den Ortsgemeinden eingebunden sind, vor allem in Bereichen, in denen es viele Entbehrungen gibt und die Herausforderungen und Chancen besonders groß sind." 
Prof. Michael BeinTKER sprach vor allem über die ,Gestaltung der akademischen Ausbildung“ in der Theologie. In den Vorbemerkungen zu seinem Beitrag orientierte Prof. BeINTKER über die theologische Aus- und Weiterbildung im Bereich oder in Verbindung mit der EKD. In Deutschland, ,findet man an 19 Universitäten Evangelisch-Theologische Fakultäten bzw. Fachbereiche. Darüber hinaus gib es drei staatlich anerkannte wissenschaftliche Hochschulen in kirchlicher Trägerschaft."

In seinem Referat behandelte Prof. BeINTKER die folgenden Fragen: 1. Anforderungen an die Absolventen einer theologischen Ausbildung im 21. Jahrhundert; 2. Das Studium der Theologie als erste Phase der Ausbildung; 3 . Was sollte verändert werden?; und 4. Theologie an der Universitas litterarum. Nach grundlegenden und kritischen Überlegungen über den Stand und die Perspektiven der theologischen Ausbildung in Deutschland heute bemerkte der Referent, dass gerade für die Theologie das wissenschaftliche Niveau sehr anspruchsvoll ist, betonte aber, dass der Anspruch auf Wissenschaftlichkeit doch auf existentielle Rückkoppelungen angewiesen ist. ,Würden diese Rückkopplungen ausbleiben, so würde der Charakter der Theologie als einer auf die Kirche und den Glauben bezogenen Wissenschaft von den Studierenden schwerlich noch nachvollzogen werden können. Eine Theologie, die sich auf die Kirche bezogen weiß und sich der intellektuellen Vergewisserung der zentralen Fragen des Glaubens annimmt, wird unweigerlich spirituelle Dimensionen berühren und einen eigenen spirituellen Charme entfalten."

Schliesslich erwähnte Prof. BeINTKER die ökumenische Dimension der theologischen Ausbildung und wies darauf hin, dass es an den deutschen Universitäten, wie auch an anderen Universitäten in Europa, es neben den Evangelischen auch andere Theologischen Fakultäten gibt. „Die Existenz konfessionsverschiedener Fakultäten an derselben Hochschule“ - betonte Prof. BeINTKER - ,stellt eine wertvolle ökumenische Bereicherung und Chance dar. Hier kann das ökumenische Lernen unmittelbar als ,ökumenisches Studieren ' eingeübt werden.“

Die lebendige Diskussion, die auf die Referate folgte, brachte viele Gemeinsamkeiten hinsichtlich der theologischen Aus- und Weiterbildung in den verschiedenen Ländern und Kirchen Europas zum Ausdruck. Doch dabei tauchten auch Verschiedenheiten auf, die zum Teil konfessionell, aber auch kulturell wie auch wirtschaftlich bestimmt sind. Ein Aspekt dieser Verschiedenheit besteht in der Frage der Beziehung zwischen Theologie und Universität, die nicht zuletzt von der Frage der Beziehung zwischen Kirche und Staat in einem gegebenen Kontext bestimmt ist. Ein anderer Aspekt dieser Verschiedenheit besteht in der Beziehung zwischen Theologie als Studienfach und der Kirche. Ein weiterer Austausch zwischen den Theologischen Fakultäten in Europa auf eine engere Zusammenarbeit unter einander hin, erwies sich in Graz als notwendig. 


\section{Die Workshops}

Das Thema der Konsultation in Graz wurde weiter durch die folgenden fünf Workshops vertieft:

1) Ökumenische Aus- und Weiterbildung.

2) Geschlechtsspezifische Fragen in der theologischen Ausbildung.

3) Gemeinsame Forschungsprojekte.

4) Qualitätssicherung in der theologischen Ausbildung (Aspekte der Akkreditierung).

5) Strukturen akademischer Grade.

Jedem Thema der fünf Workshops wurde je anderthalb Stunden gewidmet. Während der ersten dreissig Minuten wurden zuerst in die Themen im Plenum eingeführt. Danach wurden die betreffenden Themen in vier Arbeitsgruppen besprochen. Anschliessend wurde über die Gespräche in den Arbeitsgruppen im Plenum zusammenfassend berichtet.

\section{1. Ökumenische Aus- und Weiterbildung}

Pfr. Dr. Günter WASSERBERG aus Hannover, Deutschland, sprach in seiner Einführung zu diesem Workshop über „Grundsätze ökumenischen Lernens“:

1. Die eigene christlich-konfessionelle Identität setzt als erstes profunde Kenntnisse über die Geschichte und das Wesen der eigenen Konfession voraus. Ich muss wissen, wer ich - konfessionell - bin, aus welcher Tradition meine Konfession kommt und welches die Eckpunkte meiner Konfession sind. Daraus erwächst die Kenntnis, welchen Beitrag meine Konfession in die Ökumene einbringen kann. Diese Kenntnisse sind mehr als bloßes Wissen. Sie erfordern zugleich eine spirituelle Kompetenz, so dass ich meinen (konfessionellen) Glauben überzeugend und glaubwürdig vertreten kann.

2. Ökumenisches Lernen weiß um den Reichtum der eigenen Tradition, bleibt aber dabei nicht stehen, sondern achtet und schätzt den Wert anderer christlicher Konfessionen. Ich muss über den Anderen Bescheid wissen. Dies nicht allein aus Büchern und/oder über Dritte, sondern aus deren eigener Sicht. Wenn ich etwas über z.B. den Reichtum der orthodoxen Kirchen und Traditionen wissen will, dann frage ich am besten bei Orthodoxen nach. Thre Sicht muss ich kennen, um mir ein angemessenes Bild über den Reichtum der orthodoxen Tradition machen zu können.

3. Diese beiden Grundsätze haben als logische Konsequenz die Notwendigkeit ökumenischer Begegnung. Ökumenisches Lernen vollzieht sich in der Begegnung. Deshalb sind ökumenische Austausch- und Studienprogramme erforderlich: 
- Stipendien für Studierende,

- Austauschprogramme für Lehrende in Forschung und Lehre,

- Ökumenische Lernmodule für Studierende,

- Verankerung von ökumenischem Studienaustausch in Studien- und Prüfungsordnungen.

Wer die Charta Oecumenica ernst nimmt, muss sagen: „Es gibt keine gute Theologin, keinen guten Theologen, wenn sie oder er nicht ökumenische Theologen sind! Das sind wir der Geschichte der Kirchen in Europa schuldig. Das ist unser Auftrag und unsere Verheißung."

Zum Schluss seiner Darstellung formulierte Pfr. Dr. WASSERBERg die folgenden Fragen zur Diskussion in den Arbeitsgruppen:

- Erfahrungsaustausch: Wo haben Sie ökumenisches Lernen am eigenen Leibe erfahren?

- Entwurf eines ökumenischen Lernmoduls: Wie müsste es inhaltlich aussehen? Wie organisatorisch? Frage von Dauer, Teilnehmern und Ort. Wie sollte so ein ökumenisches Lernmodul in den Prüfungscurricula verortet werden? Pflicht- oder Kürprogramm? In welcher Phase theologischer Ausbildung sollte es stattfinden?

In den Berichten aus den Arbeitsgruppen wurden zum Thema des ersten Workshops etwa die folgenden Bemerkungen gemacht:

1. Ökumenisches Lernen geschieht durch persönliche Begegnung und Kommunikation. Dies hängt jedoch von der persönlichen Situation ab, aber auch vom soziologischen Umfeld, in dem man lebt. So besteht z.B. ein grosser Unterschied zwischen Westeuropa, wo ein allmählicher Säkularisierungsprozess stattgefunden hat, und beispielsweise Lettland, wo es während der kommunistischen Zeit durch gewaltsame Zerstörung jeglicher religiösen Identität praktisch zu einer tabula rasa gekommen ist.

2. Ökumenisches Lernen und Lehren geschieht im Rahmen der eigenen Tradition und sollte auch dort geschehen, doch Traditionen sind dynamisch und nicht statisch. Traditionen verändern sich auch durch ökumenische Kontakte ständig. Ökumenische Kontakte verändern die eigene Tradition und beeinflussen die anderen christlichen Traditionen. Dies ist nicht negativ, sondern eine Bereicherung.

3. Ökumenisches Lernen heisst nicht, etwas über Ökumene zu lernen. Das ganze theologische Studienprogramm sollte eine ökumenische Dimension haben.

4. So wichtig Lehrbuchwissen auch sein mag, es reicht als solches nicht aus. Ein Lernmodul ist kein Ersatz für Lebenserfahrungen. 
Eine andere Arbeitsgruppe hatte als Anlässe und Orte ökumenischen Lernens genannt:

a) der lebensweltliche Kontext in gemischt konfessionellen Kulturen über Nachbarschafts- oder Schulkontakte; dabei wurde das MinderheitsMehrheitsgefälle als wichtige Herausforderung thematisiert;

b) Begegnungen und Austausch im Jugendalter auf eigne Initiative wie z.B. in Taizé;

c) die politischen Ereignisse und Konstellationen wie etwa in Griechenland oder Bosnien, was auch zu interreligiösen Begegnungen und Lernprozessen (mit dem Islam) führte; die Gruppe beschloss daher, nicht nur das innerchristlich-ökumenische Lernen zu thematisieren;

d) der akademisch-berufliche Bereich, wobei der Existenz von zwei verschiedenen konfessionellen Fakultäten besondere Bedeutung beigemessen wurde;

e) offizielle Konsultationen und Begegnungen im Rahmen der ökumenischen Bewegung.

Die erste Gruppe war sich darüber einig, dass solche Lernprozesse nicht nur das bessere Verstehen der jeweils anderen ermöglichen, sondern zugleich zu einer Vertiefung des eigenen Profils führen. Als weiteres wichtiges Ergebnis solchen Lernens wurde die Differenzierung zwischen der religiös-spirituellen Ebene und den Sitten, Bräuchen, Mentalitäten etc. genannt, die einer Konfession oder Religion durch den Kulturationsprozess spezifische Ausprägungen verleihen; letztere wurden für die Verständigung als oftmals hinderlicher angesehen als die theologischen Unterschiede.

Zum Thema des ersten Workshops wurden in den Arbeitsgruppen die folgenden Empfehlungen gemacht: Jede theologische Ausbildungsstätte sollte zusätzlich zu ökumenisch ausgerichteten Studienprogrammen und Lehrplänen ein breites Angebot für Studienmöglichkeiten an Ausbildungsstätten anderer Denominationen/ Konfessionen haben.

Daher sollten in Zukunft keine ThelogieprofessorInnen berufen werden, die in ihrer Biografie keine gründlichen Erfahrungen mit ökumenischem Lernen aufweisen können. Über einen solchen Idealtyp einer ökumenisch orientierten Biografie (einer inter-ekklesialen Biografie) als Voraussetzung für eine Berufung wurde von einem Gruppenmitglied berichtet: als orthodoxer Theologe begann dieser seine Studien in Ägypten in einer islamischen Umgebung und lernte dort das Leben der koptischen Kirche näher kennen. Danach studierte er an einer protestantischen Fakultät im sehr katholischen Rom, und sein bester Freund war damals ein Jude.

Eine mono-kulturelle und mono-konfessionelle Erziehung und Ausbildung wird für den zukünftigen theologus europeus nicht ausreichen. Die Kirchen müssen mit den Bemühungen der EU konkurrieren und kostenaufwendige Programme wie ERASMUS/SOCRATES für transkulturelles Lernen entwickeln. Der Gedanke, dass der zukünftige cives europaeus mindestens drei Sprachen sprechen sollte, 
müsste als eine Herausforderung aufgegriffen werden. Können wir uns den zukünftigen theologus europaeus vorstellen als eine Person, die in der Lage ist, zumindest eine weitere konfessionelle Sprache und auch eine weitere Religionssprache und vielleicht auch die Sprache der Menschenrechte zu sprechen?

Es wurde auch erwähnt, dass das traditionelle ökumenische Lernen aus Lehrbüchern nicht mehr ausreichen wird. Ein „,empirisches“ Kennen des konfessionell anderen ist vielleicht wichtiger. Unsere Studienprogramme sollten Möglichkeiten für eine solche inter-ekklesiale Mobilität der Studierenden anbieten. Es wurde über Fälle berichtet, wo Studierende zu Feldstudien ins Ausland geschickt werden und nach ihrer Rückkehr gemeinsam darüber ein Buch schreiben müssen.

Es wurde noch über ein weiteres Beispiel für dieses praktisch-empirische Lernen berichtet, wo konfessionsverschiedene Studierende einen Monat zusammen lebten und je nach ihren verschiedenen eucharistischen Traditionen das Abendmahl miteinander feierten. Diese Lebenserfahrungen geben ihnen den Zugang zu den verschiedenen Lehren über die Eucharistie.

- Alle Fächer sollten eine ökumenische Ausrichtung haben: ökumenisches Lernen als Unterrichtsprinzip.

- Zusätzlich sollten ökumenische Studiengänge eingerichtet (schon im Bachelor-Studium) und durch Praktika ergänzt werden, um konkrete Begegnungen und Zusammenarbeit zu ermöglichen; Beispiele: eine ökumenische Kirchengeschichte; Liturgie als besonderer ökumenischer Lernort; eine ökumenische Doktorandenausbildung (wie sie dzt. bereits in Rom praktiziert wird).

- Ökumene sollte als Pflichtfach für die Prüfungen eingeführt werden, während die meisten Studienpläne dzt. Ökumene bestenfalls als Wahlfach vorsehen; Schwerpunkte: Geschichte der ökumenischen Bewegung; Kenntnis und eigenständige Beurteilung der wichtigen ökumenischen Dokumente.

- Die DozentInnen sollten in ihrer wissenschaftlichen Arbeit und ihrer Lebenspraxis Vorbilder für ökumenisches Lernen sein.

- Für die Studierenden sollten Begegnungsräume geschaffen und institutionalisiert werden wie etwa: spirituelle Begegnungen nach dem Beispiel von Taizé; interfakultäre diakonische Praktika; internationaler Austausch Studierender auf der Basis von Stipendienprogrammen (für mindestens ein Jahr); der Gruppe war allerdings bewusst, dass das Sprachproblem ein Hindernis darstellen kann bzw. den Studierenden ein besonderes Engagement abfordert.

- Eine Schulung in Schlüsselqualifikationen sollte eingeführt werden, damit die Studierenden persönliche Kompetenz in Kommunikation erwerben, um interkonfessionelle und interreligiöse Dialoge nach reflektierten Kriterien führen zu können. 


\subsection{Geschlechtsspezifische Fragen in der theologischen Ausbildung}

In der Einführung zum zweiten Workshop hatte Prof. Dr. Anne Kull aus Estland unter anderem folgendes bemerkt: „Traditionsgemäss hat sich die Theologie auf vier Quellen berufen: Schrift, Tradition, Erfahrung und Vernunft. Jeder Theologe/ jede Theologin benutzt diese Quellen auf unterschiedliche Weise: Manchmal denkt sie/er, streng schriftgemäss vorzugehen, während sie/er sich tatsächlich mit einem Aspekt der Tradition befasst, und umgekehrt. Die Rolle der Erfahrung ist heute besonders umstritten. Es ist immer sinnvoll zu fragen: Geht die Theologin davon aus, dass ihr Material ausserhalb der menschlichen Erfahrung liegt (z.B. die göttliche Transzendenz)? Oder identifiziert sie ihre Quellen als Erfahrung? Oder gebraucht sie Erfahrung auf eine andere Weise? Welche Rolle spielt die Subjektivität in ihrer Arbeit? Glaubt sie, dass sie sich als Wissende und Glaubende von dem trennen kann, was sie als ihr Vorgegebenes studiert?"

Wenn Erfahrung und Religion miteinander in Verbindung gebracht werden, treten geschlechtsspezifische Fragen in den Vordergrund. Diese Fragen sind zugleich global und lokal. Es sind auch kontroverse Fragen - trotz der Tatsache, dass der Feminismus als eine kritische Methode, Sichtweise und Einstellung bereits eine lange Tradition hinter sich hat. Dass es sich um eine lokale Frage handelt, wird deutlich, sobald man sich über die unterschiedlichen Probleme in den verschiedenen Staaten Afrikas, Asiens, der amerikanischen Kontinente und Europas Gedanken macht. Selbst unter den skandinavischen Ländern oder den kleinen baltischen Staaten gibt es beträchtliche Unterschiede. Geschlechtsspezifische Fragen werden mit geprägt durch Erfahrungen von Kolonialismus, Besatzung, Armut, Krieg, Globalisierung, Rassismus und Sexismus. Sie haben sehr viel mit der Frage der Identität zu tun. - Was/wo ist unsere Identität als ChristIn, KatholikIn, LutheranerIn usw.? Hat Gott Lateinisch, Deutsch, Slawisch oder eine andere Sprache gesprochen?

An manchen Orten ist die offizielle Redeweise im Blick auf stärkere Berücksichtigung geschlechtsspezifischer Fragen in der Theologie natürlich ganz positiv, doch die Rhetorik entspricht nicht der praktischen Erfahrung. An der Diskussion müssen Studierende, Fakultätsmitglieder, Amtsautoritäten der (akademischen und der kirchlichen) Institutionen und die Welt, d.h. die Gesellschaft beteiligt werden .

Auch steigt die Zahl der Frauen, die in der Kirche arbeiten werden, nicht so auffallend an wie die Zahl der Studierenden - obwohl der Pfarrberuf in gewissem Sinne ein klassisch weiblicher Beruf ist: unbegrenzte Arbeitszeit, Arbeit am Wochenende, niedrige Bezahlung und niedriger Status, Dienen und Helfen als Merkmale der pastoralen Berufung.

Die theologische Ausbildung sollte auch eine Ausbildung in christlicher und nicht ausschliesslich kirchlicher Spiritualität sein. Die AbsolventInnen der Universitäten sind oft nicht in der Lage, eine religiöse Sprache zu sprechen, oder wenn sie es tun, dann auf einer völlig unreflektierten Ebene und ohne innere Überzeugung. Die Forderung von mehr Gleichberechtigung und Partizipation für Frauen gründet 
letztlich auf biblisch-theologischen Einsichten. Es kann nicht sein, dass das Evangelium eine Botschaft der Befreiung für einige und im Bezug auf andere Fragen oder andere Personen etwas anderes ist.

$\mathrm{Zu}$ den Möglichkeiten, die theologische Ausbildung geschlechtsspezifisch zu gestalten, gehören örtliche und internationale Konsultationen, die Notwendigkeit von Vernetzung, Teilen von Ressourcen, Gedankenaustausch und gegenseitige Unterstützung Fördern und Bekräftigen. Grundlegende geschlechtsspezifische Analysen erfordern keine Vorkenntnisse, da sie auf unserer geschlechtsbezogenen Erfahrung aufbauen. Eine solche Theologie befreit Männer genauso wie Frauen und lässt Männer ihre eigene geschlechtsspezifische Erfahrung erkennen. Eine solche Theologie fragt nach den nötigen Voraussetzungen: Welche sollten es sein Philosophie, geschlechtsspezifische Studien, feministische Theorien, Soziologie oder etwas anderes? Durch geschlechtsspezifische Theologie werden verlorengegangene Geschichten und Traditionen wiederentdeckt und kulturelle Stereotypen durchbrochen. Der Text der Bibel wird von seiner patriarchalischen Versklavung befreit. Geschlechtsspezifische Analyse ist zugleich eine Gelegenheit und ein Potential. Auch wo Institutionen nicht bereit sind, dieses Instrument zu nutzen, können Einzelpersonen sich doch stärker ihrer eigenen Situation und möglichen Einflussnahme bewusst werden.

\section{Sprache}

Im Zusammenhang mit geschlechtsbezogenen Fragen stellt sich auch die Frage der Sprache. Es geht um die Bestimmung der Terminologie. Durch die mangelnde Klarheit im Blick auf den ,Gender“-Begriff selbst und die Bedeutung von Begriffen wie „Feminismus“ wird die Diskussion erschwert. So ist z.B. feministische Theologie nicht dasselbe wie geschlechtsspezifische Studien (gender studies), was wiederum nicht dasselbe ist wie geschlechtsspezifisch orientierte Theologie, was wiederum nicht dasselbe ist wie Frauenordination. Uns muss an einer inklusiven Sprache im Blick auf die Menschen und im Blick auf Gott gelegen sein. Hier muss der örtliche Kontext berücksichtigt werden: eine sprachliche Form, die in einem bestimmten Kontext passt, ist in einem anderen Kontext nicht notwendigerweise angemessen.

\section{Macht}

Zur geschlechtsspezifischen Analyse gehört notwendigerweise eine Analyse der Machtverhältnisse. Gender-Analyse führt zur Analyse von Klasse und Ethnizität (das Gegenteil trifft nicht notwendigerweise zu). Dabei muss auf die politische, strukturelle und organisatorische Dynamik sowie auf Prozesse des organisatorischen Wandels geachtet werden. Wir müssen parallele Prozesse zwischen geschlechtsspezifisch orientierter Theologie und geschlechtsspezifisch orientierter 
Kirche und kirchlichen Strukturen einleiten. Dabei ist nicht nur der Ausbildungsbereich, sondern auch der Bereich der Beschäftigung nach Abschluss der Ausbildung zu beachten. Dazu gehören auch die Medien - was die Medien aussagen über Frauen, feministische Pastorinnen oder Bischöfinnen und Theologinnen.

Bislang festgestelle Hindernisse im Blick auf geschlechtsspezifisch orientierte theologische Ausbildung:

Geschlechtsspezifisch orientierte Theologie erfordert eine schwer zu erreichende Auffassungsänderung. Rein symbolische Schritte sind zwar eine positive Handlung, die aber negative Folgen hat und einen weiteren Wandel verhindert. Dies ist das gefährlichste Stadium, das sowohl die Frauen als auch die Kirche verwundbar macht.

Angst: Veränderung ist bedrohlich. Mächtige und kluge Frauen ängstigen. Eine Fragen stellende Theologie ist beängstigend. Die Verbindung von geschlechtsspezifisch orientierter Theologie mit homosexuellen/lesbischen/bisexuellen/transsexuellen Personen richtet häufig Barrieren auf, kann aber auch Möglichkeiten eröffnen. Vielleicht ist es ein gesondertes Thema, das nicht als wesentlicher Inhalt geschlechtsspezifischer Theologie verstanden wird.

Begrenzte finanzielle Mittel wirken sich auf verschiedene Weise aus: manchmal sind keine Mittel für geschlechtsspezifische Untersuchungen verfügbar. Es gibt nicht genügend Stipendienmittel, um Theologiestudentinnen zu unterstützen, die gleichzeitig ihre Rolle als Mutter und Betreuerin wahrnehmen müssen. Der Mangel an Arbeitsmöglichkeiten nach Abschluss der Ausbildung hält Frauen ausserdem vom Studium ab.

Das geschlechtlich differenzierte Denken fordert die Theologie dazu heraus, nicht vor der Auseinandersetzung mit den Grundlagen zurückzuschrecken, die androzentrische Ordnung als ganze zu betrachten und eine Gemeinschaft des Diskurses als einen Ort aufzubauen, in dem Vertrauen entstehen kann.

Der Bericht einer der Arbeitsgruppen zu diesem Workshop hatte festgestellt, dass ,die geschlechtsspezifischen Probleme unterschiedlich bewertet sind, aber nicht zwischen protestantischen Kirchen einerseits und orthodoxen und katholischen andererseits, sondern auch innerhalb des Protestantismus und auch verschieden zwischen Gesellschaft und Theologie. Die Orthodoxen haben betont, dass die Bewertung der Frau nicht auf die Ordination zugespitzt werden darf, auch wurde von orthodoxer Seite eine Anknüpfung an das altkirchlichen Diakonat der Frau für möglich gehalten. Es wurde festgestellt, dass diese Frage auch nicht mehr so aggressiv verhandelt wird als vor 20 Jahren. Es hat eine Entwicklung stattgefunden. Angesichts der Tatsache, wie die Frauen allgemein vor 100 Jahren bewertet wurden und wie es heute geschieht, ist eine Entwicklung zu verzeichnen, die ermutigend ist."

Eine andere Arbeitsgruppe beschäftigte sich zunächst mit dem gegenwärtigen Stand und stellte fest: 
a) Beruflich sind Frauen in der Kirche und an den Fakultäten immer noch in sehr geringer Zahl vertreten und stehen am Rande; dasselbe gilt auch für die Frauen- und Gender-Forschung.

b) Der Zuwachs an Frauen wurde als wünschenswert und notwendig erachtet.

c) Hinsichtlich der akademischen Ausbildung fallen Qualifikationszeit und Familiengründung zusammen, was immer noch zu einer unzumutbaren Mehrfachbelastung für Frauen führt.

d) Es wird zu wenig wahrgenommen, dass die Zusammenarbeit von Frauen und Männern, vergleichbar dem interkonfessionellen und interreligiösen Dialog, konkrete Begegnungen und persönliche Qualifikationen für einen empathischen Umgang erfordert, der Differenzen anerkennen kann.

Eine weitere Diskussionsrunde in der gleichen Arbeitsgruppe klärte den Unterschied zwischen Frauen- und Gender-Forschung und kam überein, dass beide Richtungen für die Ausbildung wichtig sind, aber für die Männer Gender-Forschung, verstanden als Reflexion der Beziehungen zwischen den Geschlechtern, für die konkrete Zusammenarbeit besondere Bedeutung hat. Sodann wurden in beachtlicher und auch selbstkritischer Offenheit Problemfelder thematisiert:

a) das Gefälle zwischen Dozenten und Dozentinnen an römisch-katholischen Fakultäten, da Frauen keine Priesterinnen sein können;

b) die Stellung von Frauen in der Orthodoxie;

c) die immer noch einseitige, nicht egalitäre Erziehung von Mädchen und Knaben besonders im christlichen Kontext;

d) das abnehmende Interesse vieler Studentinnen an Frauen- und GenderThemen;

e) die Erfahrung, dass auch dort, wo Rechtsgleichheit gegeben ist (wie in der Mehrheit der reformatorischen Kirchen und an den Universitäten), sich die negative Haltung und Einstellung von vielen Männern gegenüber Frauen nicht geändert hat;

f) die Schwierigkeit von Männern, aus ihren Mutter (Zölibat)- und Frauenerfahrungen keine einseitigen Frauenbilder zu entwickeln, um diese dann auf Studentinnen, Mitarbeiterinnen und Kolleginnen zu übertragen;

g) das Problem, dass Studentinnen bei Prüfungen meist nur Männern gegenüber sitzen, was die Objektivität beeinträchtigen kann.

Schliesslich konzentrierte sich eine dritte Arbeitsgruppe auf das Hauptthema der Stellung der Frauen - Zugang zu Studium, Lehrpositionen, Rolle der Frauen in der Kirche. Innerhalb der Gruppe wurden sehr unterschiedliche Meinungen geäussert.

1. „Einige betonten die Bedeutung der praktischen Schritte, die unternommen werden müssen, um die Beteiligung der Frauen zu fördern - Studienprogramme für feministische Theologie, Doktorandenprogramme, Lehrmöglichkeiten. 
2. Einige aus der orthodoxen Tradition begrüssten die wachsende Zahl von Theologiestudentinnen und einige Lehrmöglichkeiten. Doch das Priestertum für Frauen ist in dieser Tradition eine unmögliche Frage.

3. Eine weitere Frage ist, wie wir mit unterschiedlichen Auffassungen in diesen Angelegenheiten umgehen. Sind dies kirchentrennende Fragen, oder können wir trotz unserer Unterschiede die Einheit der Kirche bewahren?

4. Die Bedeutung unbewusster Gefühle, die verletzende Vorurteile hervorrufen, muss erkannt werden. Dazu wurden konkrete Beispiele gegeben, zum Beispiel bei der Beurteilung von Examensarbeiten - wenn das Geschlecht der Studierenden bekannt war, wurden ganz andere Noten gegeben als bei einer anonymen Beurteilung.

5. Schliesslich ging es um Fragen der Sprache. Einige wollten die Notwendigkeit unterstreichen, sich für inklusive Sprache im Bezug auf Frauen und Männern einzusetzen, hielten diese Unterscheidung aber im Blick auf Reden über Gott nicht für relevant".

\section{Empfehlungen aus den Arbeitsgruppen:}

a) Gender-studies sollten als eigenes Fach in das Curriculum integriert werden; solange das Verhältnis von Dozenten und Dozentinnen nicht einigermassen ausgeglichen ist.

b) Die Frauengeschichte mit ihren starken Frauengestalten wäre neu zu entdecken.

c) Geschlecht sollte als Begrenzung des Menschseins wahrgenommen werden.

d) Gender-studies als Lernprojekt für Dozenten: die eigenen Mutter- und Frauenerfahrungen reflektieren, nicht undifferenziert von einem ,,weiblich-natürlichen Wesen" ausgehen, sondern Frauen als individuelle Personen und deren spezifische Kompetenz beachten.

e) Es sollte eine qualifizierte Supervision der Prüfungen von Studentinnen eingerichtet werden, wenn diese Prüfungen nur von Männern geleitet werden.

f) Die wissenschaftliche Qualifikation (Dissertation, Habilitation) von Frauen mit Familie sollte durch individuelle Gestaltungsmöglichkeiten der Arbeit und finanzielle Förderung unterstützt werden.

\subsection{Gemeinsame Forschungsprojekte}

Prof. Dr. John Muddiman gab seiner Einführung in das Thema der Arbeitsgruppe 3 den Titel: „Solipsismus versus collaboration“. Theologische Forschung wird gewöhnlich als das verstanden, was der/die einzelne Wissenschaftler/in in der Freizeit allein im Studierzimmer oder in der Bibliothek tut, nachdem die mehr gemeinschaftsbezogenen Pflichten des Unterrichtens und der Verwaltung erledigt wurden. Der/die Wissenschaftler/in wählt typischerweise ein kleines entweder 
vernachlässigtes oder kontroverses Problem aus einem grösseren Studienbereich, stellt alle Primärquellen aus den jüngsten kritischen Ausgaben zusammen und durchsucht den Index publizierter Sekundärliteratur in Büchern und Zeitschriften, um einen aktuellen status quaestionis aufzustellen, bevor er/sie eine neue Lösung vorschlägt, die dann dem ständig anwachsenden offiziellen Wissenschaftsbestand hinzugefügt wird und als Beweis von Forschungstätigkeit dient und somit für kontinuierliche Beschäftigung sorgt. Persönlicher Dialog mit anderen Wissenschaftlern gilt als zweitrangig und ziemlich amateurhaft im Vergleich zu dieser abgewogenen, gut dokumentierten professionellen Arbeit. Ich nenne dies das solipsistische Forschungsmodell. In bestimmten sehr spezialisierten Bereichen, wie beispielsweise der Untersuchung einiger erhaltener Fragmente der Schriftrollen vom Toten Meer, ist dies wahrscheinlich unvermeidlich, doch es hat als vorherrschendes Modell für theologische Forschung im allgemeinen schwerwiegende Mängel.

1. Warum ist das kollaborative Forschungsmodell für die Zukunft der Theologie in Europa so notwendig?

a) Der sich beschleunigende Fortschritt in der theologischen Wissenschaft. Kein/ keine Wissenschaftler/in kann im Alleingang damit standhalten. Um am eigenen Standort zu bleiben, muss man so schnell wie nur möglich laufen, und um woandershin zu kommen, muss man noch schneller laufen. Ich kann dies für meine eigene Disziplin, neutestamentliche Studien, beschreiben: Ich gehöre zu einem E-Netzwerk über die Paulusbriefe und bekomme täglich zwischen 5 und $20 \mathrm{E}$-mails mit neuen Theorien über die Auslegung von bestimmten Textstellen. Wir brauchen gesgenseitige Hilfe, um mit dem Tempo des Wandels Schritt zu halten.

b) Die grosse Vielfalt der Sprachen und die reiche Tradition der Wissenschaft in Europa. Die Wissenschaftlicher/innen stehen in der starken Versuchung, in dem Sprachbereich zu verweilen, den sie selbst beherrschen - Englisch, Französisch und Deutsch in meinem Fall - und alle die anderen Sprachen zu ignorieren, doch der sprachliche Imperialismus der grossen Drei wird bald zu Ende sein, ganz sicherlich mit der Erweiterung der EU und der Notwendigkeit, kulturelle und somit auch sprachliche Identität im neuen Europa zu bewahren. Und doch kann kein einzelner Wissenschaftler alle diese Sprachen meistern. Wir müssen zusammenarbeiten, um die reiche Vielfalt der wissenschaftlichen Erkenntnisse auf unsere gemeinsamen theologischen Probleme anzuwenden. Ich kann mir vorstellen, dass einmal eine Zeit kommen wird, wo gemeinsam verfasste Papiere von WissenschaftlerInnen aus Ost und West und Nord und Süd in der Theologie etwas ganz Normales sein werden, wie es in den Naturwissenschaften bereits der Fall ist.

c) Lücken in den bestehenden wissenschaftlichen Netzwerken. In bestimmten Bereichen der Theologie, wie biblischen Studien und philosophischer Theo- 
logie, gibt es bereits gut entwickelte wissenschaftliche Netzwerke auf dem „world-wide web“, obwohl zweifellos noch mehr getan werden müsste, um WissenschaftlerInnen einzubeziehen, die durch politische Umstände in ihrem jeweiligen Land an einer vollen Beteiligung an diesem internationalen Gedankenaustausch gehindert wurden. In einigen theologischen Studienbereichen ist die Zusammenarbeit jedoch noch sehr schwach und unterentwickelt. Ich denke hier an Kirchengeschichte, Liturgie, praktische Theologie usw., die weitgehend in geographischer und sprachlicher Isolierung studiert werden, wenngleich die Probleme oft gemeinsamer Art sind oder zumindest von einer vergleichenden Analyse profitieren würden.

2. Die (bisherige) Vernachlässigung der Theologie in den EU-Initiativen zur Höheren Bildung. Anders ausgedrückt: ,,Theologie ist in Brüssel unsichtbar“ . Ich glaube nicht, dass dies einfach etwas mit anti-religiösem Vorurteil bei den Eurokraten zu tun hat. Wenn wir beweisen können, und es ist nicht schwierig, dies zu beweisen, dass der christliche Glaube einer der wichtigsten gemeinsamen kulturellen Faktoren ist, der Europa in Ost und West, Nord und Süd vereint, dann sollte Theologie als das rationale Studium des christlichen Glaubens eine hohe Priorität bei der politischen Gestaltung des neuen Europa haben. Das Problem ist eher organisatorischer Art. Die Naturwissenschaften haben ihre Gelegenheit schnell und effektiv wahrgenommen. Wir in der Theologie sind dabei zu langsam gewesen, da wir geschwächt sind durch Konflikte zwischen Theologie und Religionswissenschaft, zwischen beruflicher und akademischer Ausbildung, zwischen kirchlicher Autorität und akademischer Freiheit, was ja gerade die Themen sind, mit denen diese Konsultation sich befasst.

3. Externe Finanzierung ist von Zusammenarbeit abhängig. Es ist eine allgemein bekannte Tatsache, dass Nationen und europäische Finanzierungsgremien Projekte bevorzugen, an denen zwei oder mehr Universitätsfakultäten und/oder zwei oder mehr Mitgliedsstaaten beteiligt sind. Dies ist eine bewusste Politik, um gemeinschaftliche Forschung vorrangig zu unterstützen aus dem nicht unvernünftigen Grund, dass der gesellschaftliche Effekt und Wert gemeinsamer Projekte, insbesondere im Bereich der Humanwissenschaften, sie für öffentliche Mittel unterstützungswürdig macht. Ich glaube nicht, dass wir von pluralistischen, säkularen Demokratien mehr als dies erwarten können, d.h. wir können nicht erwarten, dass sie den wesensmässigen Wert der theologischen Forschung per se berücksichtigen, aber wir sollten dennoch voll von dem Gebrauch machen, was uns angeboten wird, da damit unser eigener theologischer und ökumenischer Auftrag in keiner Weise kompromittiert wird. Und damit komme ich zu meinem letzten Punkt.

4. Einheit und Kohärenz als Grundanliegen der Theologie. Theologie ist nicht einfach die Anhäufung eines komplexen und vielschichtigen Konglomerats von Ex- 
pertenwissen, das nur anderen Experten verständlich ist. Theologie hat in erster Linie etwas mit der Einheit und Kohärenz des christlichen Theismus zu tun und mit der Vermittlung von dessen Erkenntnissen und ihrer möglichst weiten Verbreitung. Für die Naturwissenschaften ist Innovation vielleicht das Hauptziel. Für die Theologie sind Profundität und Klarheit genauso wichtig, wenn nicht noch wichtiger. Obwohl es für die Theologie notwendig ist, eine gewisse Distanz gegenüber der Katechetik und der Apologetik zu wahren, ist sie ihrem eigentlichen Wesen nach genauso auf eine effektive Kommunikation bedacht wie jene anderen mehr kirchlichen Anliegen.

Aus diesen und wahrscheinlich noch mehreren anderen Gründen ist gemeinschaftliche Forschung für die Zukunft der Theologie in Europa entscheidend. In diesem Kreise habe ich das angenehme Gefühl, zu den bereits Bekehrten zu predigen.

Fragen für die Arbeitsgruppen

1. In welchen Bereichen würde es sich nach Meinung der Gruppenmitglieder am meisten lohnen, Möglichkeiten der wissenschaftlichen Zusammenarbeit zu erkunden?

2. Haben die kirchlichen VertreterInnen in den Gruppen benennbaren Forschungsbedarf, dem die Universitätsfakultäten entgegenkommen könnten?

3. Besteht Bedarf an einem europaweiten Register theologischer Forschungsinteressen? Welches wäre der geeignetste Ort für den Standort eines solchen Registers?

4. Wie kann Information über Gastdozenturen/Gastprofessuren und Studentenaustausch auf der Master-/Doktorandenebene effektiver verbreitet werden?

5. Welche Möglichkeiten gibt es für höhere akademische Grade, die von zwei oder mehr europäischen Institutionen gemeinsam bewertet werden?

6. Welche externen Finanzierungsmöglichkeiten gibt es, die von theologischen Fakultäten bislang nicht genutzt worden sind?

Im Bericht der ersten Arbeitsgruppe über diesen Workshop wurde als Ziele und Probleme benannt:

a) Es sollte an einer zentralen Stelle ein Register theologischer Forschungsprojekte aller theologischer Fakultäten angelegt und betreut sowie in einer homepage und evtl. auch in einer Broschüre regelmäßig veröffentlicht werden.

b) Die offene Frage: Wo soll dieses Büro angesiedelt werden? Wie kann ein Betreuungsteam aussehen? Wer finanziert diese Dienstleistung? 
c) Es wäre wünschenswert, mit einem solchen Register über Europa hinauszugehen. Dies würde aber den finanziellen Aufwand noch erhöhen.

d) Auch die Idee, eine entsprechende Organisationsstelle innerhalb des Fakultätentages oder in einer der beiden Wissenschaftlichen Gesellschaften für Theologie (ev. und kath.) einzurichten, stellt vor die Frage der Finanzierung.

e) Über ein Register hinaus, bedarf es des regelmäßigen kollegialen Austausches auf eigenen Forschungskonferenzen. In der Ev. Wissenschaftlichen Gesellschaft für Theologie wird das z.T. in den jährlichen Sektionstagungen zu bestimmten ausgewählten Forschungsthemen verwirklicht; aber auch hier müssen Eigenmittel eingesetzt werden, und eine Erweiterung in den ökumenischen und außereuropäischen Bereich würde die Kosten erheblich erhöhen. Außerdem bedürfte es wieder einer zentralen Organisationseinheit.

f) Offen blieb auch die Frage, wie damit umzugehen sei, dass es in der theologischen Wissenschaft keine Tradition des Fundraising gibt. Daher würde es eines darin spezialisierten Teams bedürfen, das alle Fakultäten betreut, aber wieder finanziert werden muss.

g) Selbst dort, wo einzelne Lehrstühle geförderte Forschungsprojekte betreiben, stellt sich das Problem der geringen personellen Ressourcen für die fachliche Schulung und die regelmäßige Betreuung der im Rahmen des Projekts angestellten MitarbeiterInnen.

h) Die Finanzierung von Forschungsprojekten durch die Kirchen wäre nur von eingeschränkter Bedeutung, denn: Die Kirchen haben lediglich an lokalen Forschungsthemen Interesse und würden wenn, dann nur solche in Auftrag geben.

Der Bericht einer zweiten Arbeitsgruppe betonte, dass die Forschung zum Eigentlichen der Theologie gehört. Nur durch die Forschung kann es zu einem Wandel in den Vorverständnissen kommen, die oftmals in der Geschichte auch zu Feindschaften geführt haben. Wenn kircheneigene Projekte auch von Brüssel gefördert werden, ist dies nur zu begrüssen. Aber es kann auch sehr kirchenfremd sein, was in Brüssel als Forschungsprojekt angeregt wird. Hier wurde ein grosses Unbehagen laut. Aber es bleibt eine wichtige Aufgabe der Theologie, so zu forschen, dass sich Kirchen und Theologen europaweit näher kommen und zusammenarbeiten.

Im Bericht einer dritten Gruppe wurde Folgendes bemerkt:

1. Als erster und einfacher Schritt wurde vorgeschlagen, zur Verbesserung der Kommunikation ein E-mail-Netzwerk einzurichten.

2. Wir wollten einen Schritt zurückgehen und nach dem Zweck von Netzwerken oder gemeinsamen Forschungsprojekten fragen. Es wurden einige Prioritäten vorgeschlagen - der Dienst an den Kirchen, die Förderung der Evangeliumswahrheit. 
3. Wir stellten fest, dass das Problem besteht, dass nicht bekannt ist, was heute an den theologischen Fakultären geschieht - wir müssen mehr darüber wissen, was dort bereits geschieht. Als Teil dieser Vernetzungsinitiative sollte ausserdem ein Forum für Theologie in Europa geschaffen werden (die Geldfrage ist dabei sekundär). Die theologischen Fakultäten und nicht die Kirchen sollten dabei die Initiative ergreifen, allerdings mit der Unterstützung und Partnerschaft der Kirchen. Für einige Mitglieder wäre es hilfreich, wenn das akademische Netzwerk durch die KEK koordiniert werden könnte.

4. Es wurde ein Vergleich mit der heutigen Lage in Russland gezogen. Am dringendsten ist eine Vision und geistige Unterstützung für die theologische Ausbildung, um einer sehr schwierigen rechtlichen Situation entgegenzuwirken, in der es kein Recht auf Unterricht im Glauben gibt. (Es ist lediglich auf informeller Basis ,erlaubt“.) So könnten die Argumente, dass das Netzwerk im Rahmen der EU entstehen könnte, für die russische Kirche bei den Verhandlungen mit ihrer Regierung hilfreich sein.

5. Die blosse Zahl der bestehenden Netzwerke und akademischen Partnerschaften ist verwirrend. Vielleicht sollten wir unsere Bemühungen darauf konzentrieren, bestehende Partnerschaften zu fördern, statt neue aufzubauen. (Die mit dem Aufbau von Netzwerken verbundene Arbeit kann als solche viel Zeit verschlingen, die besser für die Forschung selbst genutzt werden könnte.)

\subsection{Qualitätssicherung in der theologischen Ausbildung (Aspekte der Akkreditierung)}

Pfarrer Arne ERIKSEN aus Norwegen und Dr. Wolfgang WeIRER von der Universität Graz gaben eine Einführung in das Thema dieser Arbeitsgruppe. Pfarrer ERIKSEN begann mit folgenden Bemerkungen: Die an dieser Konsultation beteiligten Vertreter und Vertreterinnen aus dem Bereich der theologischen Ausbildung und der Vorbereitung auf das Pfarramt sollten ein wesentlicher Teil des Bologna-Prozesses sein. Hauptziel dieses Bologna-Prozesses sei es nämlich, bis zum Jahr 2010 einen Europäischen Raum für Höhere Bildung (EHEA - European Higher Education Area) zu schaffen. Meine Frage dazu ist: Welche Rolle werden die theologische Ausbildung und die Vorbereitung auf das Pfarramt innerhalb dieses EHEA spielen?

Die treibende Kraft hinter dem Bologna-Prozess war bisher eine enge Zusammenarbeit zwischen den europäischen Regierungen auf der Grundlage von Erklärungen und Dokumenten der alle zwei Jahre abgehaltenen Gipfeltreffen (Bologna 1999, Prag 2001, Berlin 18.-19. September 2003). Doch dieser Prozess wird auch von den Universitäten und ihren Organisationen stark unterstützt - koordiniert durch die Vereinigung Europäischer Universitäten (European University Association-EUA) mit ihren wichtigen Tagungen (Salamanca 2001, Graz 29.-31. Mai 2003). Auf weltweiter Ebene werden diese Entwicklungen durch internationale 
Bemühungen unterstützt, die von der UNESCO und der Welthandelsorganisation (World Trade Organisation-WTO) und ihrem Allgemeinen Abkommen über Handel bei Dienstleistungen (General Agreement on Trade in Services-GATS) unterstützt.

Die Bologna-Erklärung (1999) stellt vier wichtige Aspekte für die Feststellung von Ähnlichkeiten im Bereich der höheren Bildung in Europa heraus: Lesbarkeit, Vergleichbarkeit, Kompatibilität und Transparenz. Dies sind alles Aspekte der Qualität. Daher unterstreicht die Bologna-Erklärung die Notwendigkeit einer Förderung der europäischen Zusammenarbeit in der Qualitätssicherung, die ausgerichtet ist auf die Erarbeitung vergleichbarer Standards, Kriterien und Methoden (Bologna-Erklärung 1999). Eine der Empfehlungen des Gipfels von Bologna war, dass die beteiligten Länder Systeme für eine transparente Qualitätsbeurteilung und Qualitätssicherung entwickeln sollten.

Wichtige Partner für die europäische Zusammenarbeit im Blick auf Qualitätssicherung sind das Europäische Netz für Qualitätssicherung (European Network for Quality Assurance-ENQA) und das Netz Mittel- und Osteuropäischer Qualitätssicherungsagenturen (Network of Central and Eastern European Quality Assurance Agencies-CEE). ENQA ist ein Netz von 25 nationalen Qualitätssicherungsagenturen, und die Zahl der Agenturen nimmt ständig zu. Eine wesentliche Aufgabe der nationalen Qualitätssicherungsagenturen besteht darin, auf nationaler Ebene Qualitätssicherungssysteme mit Akkreditierung als eine von mehreren komplementären Massnahmen zu entwickeln. Eine gemeinsame Tagung von CEE und ENQA soll vom 18.-19. Oktober 2002 in Prag stattfinden.

Anschliessend stellte Dr. Wolfgang WeIRER die Frage nach einer Kriteriologie: Als theologische Fakultäten sind wir in der Regel verschiedenen Institutionen mit möglicherweise heterogenen Interessen verpflichtet. Die Kriterien von Qualitätsentwicklung und Evaluierung werden kirchlicherseits anders definiert als sie etwa in staatlichen Evaluierungs- und Akkreditierungsagenturen gelten, es stehen andere Interessen und Ziele hinter den jeweiligen Evaluierungsbestrebungen. Es stellt sich daher m.E. zwangsläufig die Frage: „Was ist unter Qualität theologischer Lehre zu verstehen?" Diese Frage wird je nach Interessenslage unterschiedlich beantwortet werden und ich meine, es wäre wichtig, dass sich die theologischen Fakultäten selbst dieser Frage stellen, bevor sie von ,,außen “ beantwortet wird. Anhand dieser Frage definieren sich auch die Ziele von Qualitätsmanagement-Prozessen:

Ziel der von der (katholischen) Kirche initialisierten Evaluierungsprozesse ist etwa vorrangig die Kontrolle, wie sehr ein Curriculum in der Anlage und Durchführung mit den einschlägigen kirchlichen Dokumenten übereinstimmt bzw. vereinbar ist. Hintergrund ist die Idee, dass ein (katholisch-)theologischer Studiengang auf der ganzen Welt in etwa gleich gestaltet sein muss; Stichwort: Kompatibilität.

Zum universitären Verständnis von Evaluierung: In der derzeitigen universitären Praxis wird unter Evaluierung von Lehre im deutschen Sprachraum vorwiegend (noch) Bewertung einzelner Lehrveranstaltungen durch Studierende verstanden, die Evaluierung von Curricula ist noch kaum implementiert. 
Kriterien, die von universitärer Seite bzw. von Seiten diverser Evaluierungsagenturen, die im Entstehen begriffen sind, genannt werden:

- Entspricht das jeweilige Curriculum den Formalkriterien (kirchliche Vorschriften, staatliches Studienrecht ...) ?

- Wird das selbstgesetzte Ausbildungsziel erreicht?

- Wie steht es um die Kompatibilität und die internationale Ausrichtung / Vergleichbarkeit?

- Welche regionalen Schwerpunkte und Profile bilden sich im Curriculum ab (ist u.U. ein Gegensatz zu oben ...)?

- $\quad$ Ein weiteres Kriterium wäre die Transparenz der Lehrinhalte: Das hat auch noch einmal wesentliche Konsequenzen für die Mobilität der Studierenden (Anerkennungsverfahren, diploma supplement ...).

- Lehrorganisation: Administration; Studierbarkeit: mehrere Angebote; wenige Überschneidungen; Zyklus von Lehrveranstaltungen.

- Prüfungsorganisation: Transparenz von Prüfungsstandards und Kriterien.

- Prüfungsmethodik: Vgl. enzyklopädisches Wissen - Transferwissen; Zueinander von Lehrinhalten - Lehrform - Prüfung.

- $\quad$ Stimmige Modularisierung (Sinnhafte Abfolge der einzelnen Module ...).

- Verzahnung innerhalb der Universität. Kooperationen.

Kriterien, die kirchlicherseits eingefordert werden

- Hinweis: katholische Perspektive:

- Treue zum kirchlichen Lehramt

- Rahmenordnung, Fächerkanon (flächendeckend, aber keine neuen Fächer)

- Kompatibilität

- Mobilität

- _organische und vollständige Synthese“ der katholischen Theologie.

Die jeweiligen Kriterien sind zur Kenntnis zu nehmen, gehören m.E. aber auch theologisch beleuchtet: Inwiefern sind die jeweiligen Kriterien kompatibel mit den Zielen theologischer Studiengänge insgesamt?

Ich möchte nur einige kleine Beispiele nennen:

- Lassen sich Kriterien wirklich flächendeckend über Studienrichtungen herunterbrechen, ist nicht auch der jeweilige gesellschaftliche und kirchliche Kontext mitzubedenken?

- Ausgangssituation theologischer Studiengänge ist derzeit die, dass Studierende mit sehr unterschiedlichen religiösen und kirchlichen Sozialisationsbedingungen an die jeweilige Ausbildungsstätte kommen - Inwiefern nimmt die Studienkonzeption darauf Rücksicht?

- Inwiefern werden Studierende - und Lehrende - als geistbegabte Subjekte 
ihres Glaubens und ihrer Glaubensgeschichte in der Konzeption theologischer Studiengänge wahr- und ernstgenommen?

- Inwiefern ermöglicht das (katholischerseits) „gesamtkirchlich“ verordnete Kriterium der „Kompatibilität“, eines weltweit einheitlichen Rahmens des Theologiestudiums, die Ausprägung lokaler und regionaler Theologien, die auch auf die jeweilige kulturelle und gesellschaftliche Situation Bezug nehmen können?

- Inwiefern birgt das Kriterium einer „Verzahnung“ der Theologie innerhalb der Universität die Gefahr, dass einzelne Fächer auch von anderen Fachbereichen wahrgenommen werden können, dass Kirchengeschichte von der Geschichte, Kirchenrecht von der juridischen Fakultät, christliche Gesellschaftslehre von der Soziologie, Religionspädagogik von der Pädagogik wahrgenommen wird und das „Theologische“ dieser Disziplinen damit unter die Räder kommt?

Als Abschluss seiner Einführung stellte Pfarrer Arne ERIKSEn die Qualitätsagentur von Norwegen vor. Am 6. Juni dieses Jahres wurde vom norwegischen Parlament im Rahmen einer "Qualitätsreform im Bereich der Höheren Bildung“ eine neue unabhängige Qualitätssicherungsagentur eingerichtet: National Agency of Educational Quality (NOKU).

- $\quad$ Norwegen hat ein System einer offiziellen Akkreditierung für den gesamten Bereich der höheren Bildung - staatlich, kirchlich oder privat - eingeführt.

- Das systematische Begleit- und Kontrollverfahren für Akkreditierung wird sich auf Qualitätsprüfungen (audits) gründen.

- Akkreditierungsbeschlüsse der Nationalen Qualitätssicherungsagentur sind endgültig, d.h. sie können weder vom Erziehungsministerium noch von irgendeiner anderen politischen Behörde geändert werden. Akkreditierung wird sich nicht mehr auf „Besitzanspruch“, sondern auf „Qualität“ gründen.

- Die Agentur wird darüber hinaus auch andere Formen der Evaluierung durchführen.

Für die Gruppenarbeit wurden die folgenden Fragen empfohlen:

- Welche Erfahrungen haben Sie mit Qualitätssicherung im universitären Kontext gemacht (aus einer institutionellen Perspektive)?

- Welche Kriterien der Evaluierung und der Qualitätssicherung sind für theologische Ausbildungsgänge geeignet, welche weniger?

- Welche Möglichkeiten der Vernetzung der theologischen Fakultäten in Europa sehen Sie, die der Etablierung eines Qualitätsmanagement-Systems dienen können? 
Der Bericht einer Arbeitsgruppe wurde etwa zusammengefasst: Der politische Druck, sich dem Bologna-Prozess anzuschliessen, ist in Europa von Land zu Land sehr unterschiedlich: Es gibt Länder in denen dieser Prozess weithin unbekannt ist, bis hin zu Ländern, die bereits Akkreditierungsagenturen für theologische Studiengänge eingerichtet und Akkreditierungen durchgeführt haben. Kritisch wurde angemerkt, dass die Gefahr besteht, dass Evaluierungsprozesse weniger die Qualität als vielmehr Quantitäten von Studiengängen erheben. Beklagt wurde u.a. auch der enorme bürokratische Aufwand und die Gefahr, dass kirchliche Qualitätskriterien nicht das ihnen zustehende Gewicht erhalten könnten.

Aus anderen Gruppen kamen folgende Bemerkungen:

1. Erfahrungsaustausch: Unsere Gruppe tauschte verschiedene Erfahrungen aus.

a) Aus dem Vereinigten Königreich wurde berichtet, dass das dortige Evaluierungssystem ausgezeichnet sei und anscheinend gut funktioniere.

b) Einige sprachen von den schwierigen Erfahrungen, die sich aus den Nachwirkungen der kommunistischen Zeit ergeben. So gab es z.B. in Bulgarien 1989 vier Minoritäten, während es jetzt über 40 gibt, was Probleme für die Qualität mit sich bringt.

c) Einige befürchten, dass Professoren zuviel Zeit für die Vorbereitung und Durchführung der Evaluierung aufbringen müssten.

2. Die Verschiedenartigkeit der Erfahrungen innerhalb Europas deutet darauf hin, dass eine gewisse Standardisierung notwendig sein wird. Unterschiedliche geschichtliche Entwicklungen erfordern ein unterschiedliches Tempo bei der Standardisierung.

3. Es wurde darauf hingewiesen, dass ähnlich wie Finanzierungsgremien und Qualitätssicherungsagenturen als Puffer zwischen Staat und Universitäten wirken, ein entsprechender Puffer zwischen theologischen Fakultäten und den Kirchen notwendig sein könnte.

4. Es wurde auf den Mangel an finanziellem Ansporn im Blick auf den Qualitätssicherungsprozess hingewiesen. Die westeuropäischen Regierungen wollen hohe Qualität und auch mehr Quantität, sind aber nicht bereit, zusätzliche Finanzmittel bereitzustellen.

5. Es wurde ein Vergleich mit den Vereinigten Staaten angestellt, wo verschiedene Institutionen ihre eigenen Qualitätskontrollagenturen haben.

6. Es wurde die Befürchtung zum Ausdruck gebracht, dass die freiwillige Konver- 
genz von Bologna schliesslich auf gesetzliche Kontrolle und Zentralisierung hinauslaufen könnte.

Abschliessend wurde festgestellt, dass Qualitätsakkreditierung eine entscheidende Rolle dabei spielte, dass vor kurzem der theologische Fachbereich an den staatlichen Universitäten in Russland als Fakultät aufgenommen wurde.

\subsection{Strukturen akademischer Grade}

Die Einführung in den letzten Workshop wurde von Prof. Dr. Juraj B ANDY aus der Slowakei gemacht. In seiner Darstellung ging Prof. BANDY von der Überzeugung aus, dass auf dem Weg zur Kompatibilität zwischen den verschiedenen Strukturen akademischer Grade in den theologischen Fakultäten zuerst nötig ist:

(a) die verschiedenen Systeme zu vergleichen und

(b) die Kriterien, die zum Erlangen eines gewissen Grades oder Titels notwendig sind, zu vergleichen.

Man kann hier das System, das zur Zeit in der Slowakischen Republik gültig ist, als Anstoss zur Diskussion darstellen, fuhr Prof. BANDY fort. Ein Studiengang an der Universität oder an der Hochschule, der in der Regel 6 Semester dauert, endet mit dem Titel Baccalaurius (Bc).

Fragen: Sollen die Studenten der Theologie nach dem dritten Jahrgang automatisch den Titel Baccalaurius bekommen? Soll eine Art von Baccalarius Studiengang neben dem Magister - Studiengang an den theologischen Fakultäten existieren? Was kann ein Baccalaurius der Theologie im kirchlichen Dienst tun? Wofür ist der Absolvent des Baccalarius-Studiums kompetent?

In der Slowakei haben wir das Baccalaurius-Studium nicht eingeführt (obwohl angeblich die EU dafür plädiert), weil wir nicht wissen, was ein Absolvent eines solchen Studiums in der Praxis anfangen könnte. Das Studium der Theologie dauert fünf bzw. sechs Jahre (röm.-kath.). Nach dem Abschluss des Studiums bekommt der Absolvent den Titel Magister (Mgr.). Ich vermute, dass es überall so ist, wo die Theologie ihren Ort an der Universität hat. Das Problem beginnt in der Slowakei mit dem postgradualen Studium. Die römisch-katholischen Fakultäten haben nach der Inkorporation in die Universitäten nach der Wende im Jahre 1989 den Gradus Licenciatus eingeführt (Th.Lic.).

Fragen: Ist dieser Gradus notwendig? Sollten auch die nichtkatholischen Fakultäten diesen Gradus einführen? Sollte die Person, die schon einen höheren Gradus erreicht hat, weiterhin diesen Titel vor ihrem Namen schreiben?

Das nächste Problem hängt mit dem Titel Th. Dr. zusammen. In der Slowakei sind die Absolventen der Medizin automatisch MUDr., die Absolventen des juristischen und pädagogischen Studiums sind Magistri, aber nach einer zusätzlichen (nicht sehr anspruchsvollen) Prüfung erlangen sie den Titel JUDr. bzw. PaedDr. In diesem Zusammenhang hat der Titel Th.Dr. in den Augen der Öffentlichkeit keinen besonderen Wert. Es scheint so zu sein, als ob es um einen ,kleinen“ Doktortitel ginge. 
Fragen: Soll der Titel Th. Dr. abgeschafft werden? Soll dieser Titel mit dem Ph. D ersetzt werden? Soll der Titel Th. Dr. auf das Niveau des Paed. Dr. sinken? Im Zusammenhang mit der Dozentur und der Professur gibt es eine gemeinsame Frage: die Vereinheitlichung der Kriterien. Ich denke, dass ich anhand des slowakischen konkreten Kontexts genug Fragen aufgeworfen habe, die für unsere Diskussion nützlich sein könnten.

Der Bericht aus einer Arbeitsgruppe zum Workshop 5 betonte, dass es interessant war, von der Vielfalt akademischer Titel und ihrer unterschiedlichen Bedeutung in den verschiedenen Ländern zu erfahren. Zum Problem wird diese Vielfalt, wenn es um einen beruflichen Wechsel von einem Land in ein anderes geht. Insofern ist es zu begrüssen, wenn im Zuge des Bologna-Prozesses ein gemeinschaftliches System akademischer Titel geschaffen wird. Andererseits entstehen durch das dreistufige System (BA, MA, DR) verschiedene Fragen. Der BA-Abschluss soll der erste berufsqualifizierende akademische Abschluss sein. Doch für welche berufliche Tätigkeiten soll er qualifizieren bzw. ist eine berufliche Qualifizierung nach sechs Semestern überhaupt möglich? Unterschiede wurden deutlich im Verständnis der Zuordnung von BA und MA nach „Bologna“: Geht es um eine Vertiefung des BA-Studiums durch den MA-Abschluss oder um zwei verschiedene voneinander unabhängige Studiengänge? Berichtet wurde auch von den Problemen, die durch den politischen Druck zur Einführung dieses Systems entstehen (Österreich) oder durch die in Deutschland beabsichtigte Regelung, nur 20\% der Basis zum MA-Studiengang zuzulassen (Konflikt mit dem Grundrecht auf Berufsfreiheit).

Die Diskussion in einer anderen Gruppe befasste sich mit folgenden Problemen:

1. Das Problem der Bedeutung von ehrenhalber verliehenen akademischen Graden.

2. Probleme des Baccalaureus-Grades:

- in Lettland - ein Extrajahr für Sprachstudien, für das die Studierenden bezahlen müssen, ist notwendig.

- Was können wir mit einem Baccalaureus-Grad in Theologie anfangen?

3. Problem der unterschiedlichen Situationen in Europa, daher die Wichtigkeit dieser Diskussion.

4. Problem der (in den Bologna-Dokumenten nicht erwähnten) Annahme, dass die Zulassung zur Universität in allen Ländern gleich sei. Daher scheint sich die Diskussion am anderen Ende des Prozesses zu befinden.

5. Das Problem der Verwässerung der Bedeutung der Doktorate, indem man zum Beispiel einen Doktor in Kosmetik und einen Doktor in Theologie erhalten kann. Diese Entwicklung scheint von polytechnischen Ausbildungsstätten angetrieben zu werden.

Schliesslich verglich eine dritte Gruppe die Struktur der akademischen Grade in den verschiedenen hier vertretenen Ländern; keine war mit den Vorschlägen von Bologna $(3+2+3)$ identisch: 
- Schweden: 4 Jahre Theologie-Kandidatenstatus für Examen

3 Jahre Licentia, die Teil des Doktorandenkurses von mindestens 4 Jahren werden kann

- Litauen: $\quad$ B.Th in Theologie 5 Jahre (2 Jahre Philosophie, 3 Jahre Theologie)

Licentia 2 Jahre

Doktor der Theologie 4 Jahre

- Griechenland: Licentiat 4 Jahre

Magister 2 Jahre

Doktorat 3 Jahre (weitere Anforderungen für Berufung auf eine Professur)

- Rumänien: $\quad$ BA 4 oder 5 Jahre

Magister 2 Jahre

Doktorat 3-7 Jahre

- Norwegen: BA/Magister 5 Jahre, aber 6 Jahre für berufsorientierte Grade

- Schottland: MA 5 Jahre

- Estland: $\quad$ BA 3 Jahre

Magister 2 Jahre

Ph.D oder Doktor der Theologie 4 Jahre.

Es wurde weiter angemerkt, dass der erste Grad (wie immer er bezeichnet wird) in der Regel mindestens ein Jahr länger braucht als der folgende Grad und dass der Doktorgrad mindestens drei Jahre weiterer Studien erfordert. Wir haben nicht über die Gründe für die Unterschiede in der Länge der Vorbereitungszeit auf den ersten Grad gesprochen, doch dies mag etwas mit dem erreichten Niveau am Ende der Oberschule oder mit dem Mass der Spezialisierung zu tun haben.

1. Einige Länder verlangen weitere Qualifizierungen über das Doktorat hinaus (Habilitation) für die Berufung auf einen akademischen Posten, andere tun dies nicht.

2. Die D. Min (nach professioneller Erfahrung) Qualifizierung, die amerikanischer Praxis folgt, wurde als ein neuer Faktor in bestimmten Ländern (z.B. UK) erwähnt.

3. Einige Länder verleihen einen regulären $\mathrm{Ph}$. D für eine auf einen Doktorgrad zielende Arbeit in Theologie, als Hinweis auf einen erreichten gleichen Standard; andere ziehen es vor, bei Doktoraten in Theologie eine gewisse Unterscheidung vorzumehmen (D. theol.).

4. Einige wenige Länder haben noch nicht das ECTS-System für höhere Grade übernommen. 


\section{Perspektiven}

Die Konsultation der theologischen Fakultäten in Graz zielte darauf hin, durch Referate, Diskussion und Austausch eine gute Plattform für eine nähere und mehr strukturierte Zusammenarbeit zwischen den theologischen Fakultäten von ganz Europa zu schaffen. Am Ende der Tagung wurde eine Schlusserklärung angenommen, die zum Ausdruck brachte, dass auf dieser Konsultation ,,die Notwendigkeit und der Wert einer verstärkten, Länder und Konfessionen übergreifenden Kooperation der Theologischen Fakultäten, Hochschulen und Institute sichtbar wurde". In der Grazer Erklärung wurde weiter unterstrichen: „Der Austausch von Studierenden und Lehrenden und die Integration des ökumenischen Lernens in die theologische Ausbildung sind für die Zukunft der Kirchen Europas unverzichtbar. Die Erörterung ökumenischer Fragen wie auch das bessere gegenseitige Kennenlernen der verschiedenen Kirchen und Konfessionen - und zwar in der Perspektive ihrer eigenen Wahrnehmung - wurden als unerlässlich betrachtet. Es können nicht nur einzelne ökumenische Spezialveranstaltungen angeboten werden, vielmehr soll der ökumenische Gedanke alle Lehrveranstaltungen befruchten und durchdringen. Für die Förderung des ökumenischen Anliegens sind Begegnungen und Studienreisen sehr wichtig. Im zusammenwachsenden, neuen Europa soll eine gemeinsame; wenn auch nicht uniforme, christliche Stimme hörbar werden. Die theologischen Ausbildungsstätten können zu den aktuellen ethischen und sozialethischen Fragen bedeutende Impulse geben und ihren Beitrag auch im Aufzeigen von Handlungsmöglichkeiten leisten.“

Schliesslich empfahl die Konsultation einige konkrete Schritte für die Fortsetzung und Förderung einer intensiven Zusammenarbeit: ,die regelmäßige Einberufung von gesamteuropäischen Konsultationen zu Fragen der Theologie und der theologischen Ausbildung im Abstand von zwei bis drei Jahren, das Eingehen von Partnerschaften zwischen den verschiedenen Ausbildungseinrichtungen unabhängig von den jeweiligen konfessionellen Bindungen und schließlich als langfristiges Ziel die Schaffung einer Ökumenischen Konferenz Europäischer Theologischer Fakultäten, Hochschulen und Institute. Eine solche intensive Zusammenarbeit kann für die Zukunft der Gemeinschaft der Kirchen und der Völker Europas einen wertvollen Beitrag leisten.“

Die Konsultation in Graz hatte eindeutig gezeigt, wie wichtig es ist für die theologischen Fakultäten verschiedener theologischer Tradition, in einen offenen und direkten Austausch zu treten. Alle theologischen Fakultäten von ganz Europa setzen sich mit vielen Herausforderungen auseinander, die sie allein nicht mehr bewältigen können. Die Zukunft der Theologie in Europa wird entscheidend bestimmt von der Zusammenarbeit zwischen allen theologischen Fakultäten über die kulturellen, politischen, wirtschaftlichen und sogar konfessionellen Grenzen hinaus. In Graz wurde ein bedeutender Schritt in diese Richtung getan. 


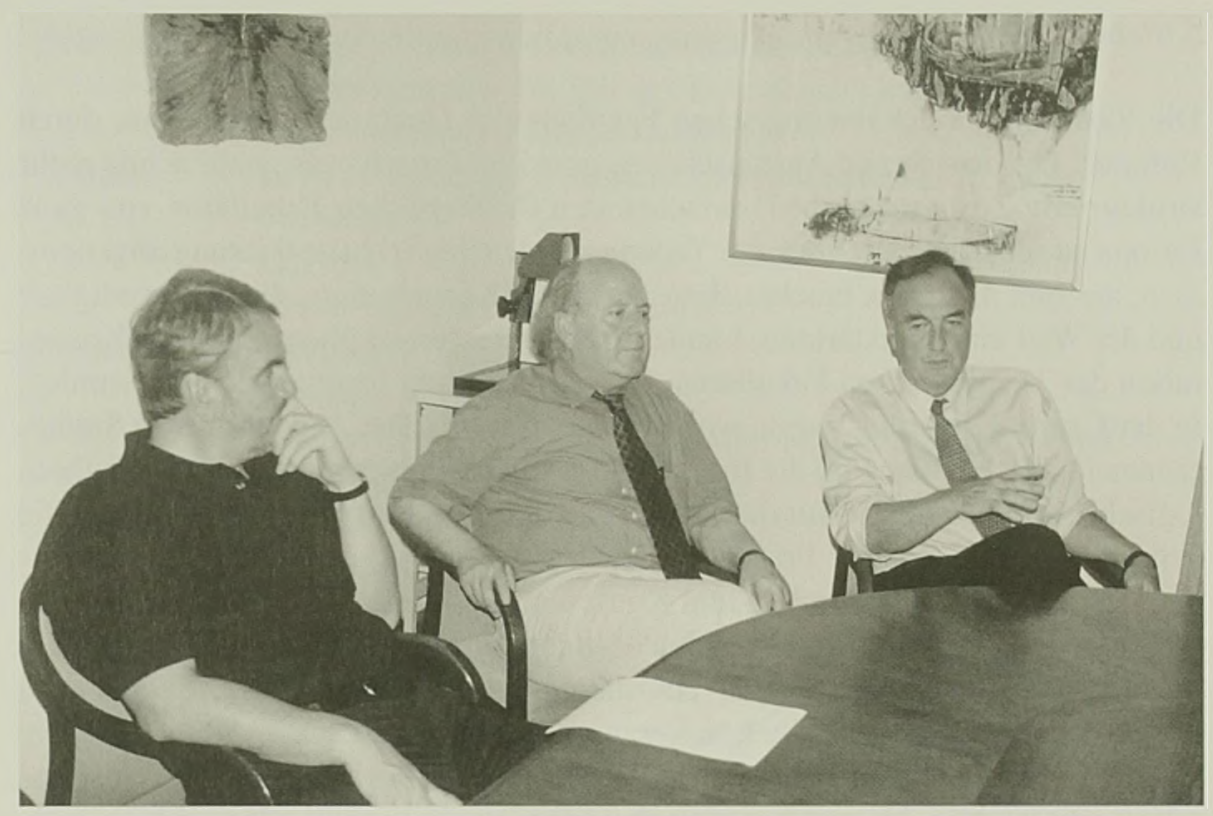

Abb. 2: (v.l.n.r.) Viorel IonItA, Studienleiter der KEK; Grigorios LARENTZAKIS, Prof. für orthodoxe und ökumenische Theologie, Graz; Gerhard LaRCHER, Dekan der Katholisch-Theologischen Fakultät, Graz 